\title{
Knowledge Systems and Risk Management: Towards a Risk and Threat Assessment Framework
}

\author{
Murray E. Jennex \\ San Diego State University \\ mjennex@sdsu.edu
}

\author{
Alexandra Durcikova \\ University of Oklahoma \\ alex@ou.edu
}

\begin{abstract}
Knowledge is the most important asset that a company can have. Thus, it is imperative that this asset is safeguarded just like generic information assets. However, knowledge management (KM) and knowledge systems are different than traditional information systems with different threats and different operational requirements. Information Security professionals recognize that risk assessment is the cornerstone to information security. We build on this perspective and propose that risk assessment techniques need to be applied to KM too to properly safeguard this asset. We discuss risk assessment frameworks and build on a KM/knowledge system specific risk assessment framework with a step-bystep guideline for $\mathrm{KM} / \mathrm{knowledge}$ system specific threat assessment.
\end{abstract}

\section{Introduction}

Whitman and Mattord [33] quote Sun Tzu Wu on the importance of knowing yourself and knowing your enemy as a key to success in battle, or in designing and implementing information systems security. To accomplish the knowing of yourself and your enemy the corner stone of information systems/cyber security is the process of risk assessment. Risk assessment is used to know yourself by identifying data/information/ knowledge/technology assets (henceforth simplified to knowledge assets) and assigning. Risk assessment helps organizations know the enemy by determining the threats that could attack the organization's knowledge assets. Risk management then uses these inputs to analyze the overall risk to the knowledge asset and determine the controls to be used to mitigate or remove the risk. All major security frameworks include a risk assessment and management process. For the United States the
Nationals Institute of Standards and Technology (NIST) provides the risk management framework (RMF) as described in special publication, SP 800-12 rev 1, An Introduction to Information Security [21], SP 800-37 rev 2, Risk Management Framework for Information Systems and Organizations: A System Life Cycle Approach for Security and Privacy [23], and SP 800-53 rev 5 (draft), Security and Privacy Controls for Information Systems and Organizations [24]. A similar process is outlined by the International Standards Organization (ISO). ISO 27001:2013 Information technology -- Security techniques -- Information security management systems - Requirements [7] and ISO 7005:2018, Information technology -- Security techniques -Information security risk management [8].

While these are fine risk frameworks, they are generic in nature and are not tailored specifically to knowledge management, KM, or knowledge systems. We do not claim that $\mathrm{KM} / \mathrm{knowledge}$ systems are so unique as to require their own risk frameworks or that the above-mentioned risk frameworks are not useful. We are stating that $\mathrm{KM} / \mathrm{knowledge}$ system managers will do a better job of risk assessment/ management of $\mathrm{KM} / \mathrm{knowledge}$ systems if they have tailored guidance, specifically in two areas: threat assessment and risk analysis. Why do we think KM/knowledge systems need special guidance? We argue that the purpose of $\mathrm{KM}$ and knowledge systems are to support the sharing and application of knowledge by supporting decision making, throughout the organization to achieve organizational goals. Since the purpose is to share knowledge the tenets of information security are inherently at odds with $\mathrm{KM} /$ knowledge systems. It is our opinion that information security is still needed but should be applied in ways that recognize the uniqueness of knowledge sharing and decision processes. Jennex and Durcikova [18] examined the integration of KM and security and found that security was not integrated into the KM job functions. Thus, the purpose of this paper is to provide specific guidance 
and requirements for $\mathrm{KM} / \mathrm{knowledge}$ systems threat assessments and risk analysis. In addition, this paper is focusing on the risk of knowledge loss from a human source (we will only focus on this asset). Knowledge loss risk is defined as the expected impact to the organization resulting from the loss of a particular expert or knowledge worker. This is consistent with the NIST [23] risk definition so the NIST risk algorithm will be used as the basis for determining knowledge loss risk.

This paper first starts with an overview of risk and risk management, threats and threat assessment, risk frameworks, and knowledge management (KM)/knowledge systems risk frameworks. This is followed by a KM/knowledge systems specific threat assessment that is based on literature review followed by a KM/knowledge systems risk framework proposal also based on literature review that incorporates the $\mathrm{KM} / \mathrm{knowledge}$ systems specific risks. The literature review includes analysis of previous case studies and other research.

\section{Background: Risk and Risk Assessment}

The NIST SP 800-37r2 [23] describes risk as the net negative impact of the exercise of a vulnerability; considering both the probability and the impact of occurrence. Risk is traditionally represented by the following formula:

$R($ risk $)=p$ (probability of occurrence) $x$ $C$ (consequence of occurrence either represented by some value or by a loss function)

Risk management is the process of identifying risk, assessing risk and taking steps to manage risk by reducing risks to an acceptable level [23]. Acceptable level of risk depends on the managers of a organization. For example, for organization A a loss of $\$ 30,000$ might be huge risk while for an organization B this amount represents a minor risk. Additionally, Smith et al. [28] and Aubert et al. [2] argue that information systems managers and researchers traditionally define risk in terms of negative consequences describing risk as the possibility of loss or damage and the possibility of suffering harm or loss.

An alternative view by Billington [3] points out that, when examined closely, "risk" can actually lead to both positive and/or negative consequences. In any particular initiative, he notes, the risks involved could represent different meaning to an organization. Billington [3] proposes three dimensions of a risk:
1. A hazard that must be minimized or eliminated;

2. An uncertainty about which path should be taken and which must be studied to reduce the variance between anticipated outcomes and actual results; and

3. An opportunity for growth or improvement, which must be assessed to determine how much innovation, initiative and entrepreneurship should be exercised.

Viewing risk as something more than a hazard is highly applicable to risk management in KM [19]. Although KM risks can lead to negative results, they can also represent significant opportunities for savings or business development. For example, losing a subject matter expert who has $30+$ years of experience in a particular aspect of a business (due to retirement) might be perceived as a huge risk that can potentially cause a large financial loss. However, this same scenario might open doors to hiring new talent that costs less money (e.g., lower starting salary) that can bring new ways of addressing business problems.

Uncertainty associated with knowledge use, be it due to rapidly changing technology and storage media, to misuse or new and unexpected uses of knowledge or to the basic understanding of the captured knowledge, is one of the biggest challenges a KM manager faces. This is the reason why this paper is focusing on the risk of knowledge loss from a human source, for example, loss of knowledge from particular expert or knowledge worker.

A final note on the risk formula shown above is that it recognizes that determining exact probabilities and values for consequence is difficult. An alternative to probabilities and consequence values proposed by several sources is to utilize relative ranges or scores for the probability and consequence value. When this is done, probability becomes likelihood and consequence value becomes consequence. This paper, as discussed later, uses this approach of utilizing scores and thus uses likelihood and consequence. This also allows a group of employees to agree on a likelihood and score rather than for one employee to come up with these values. While the scoring approach does not provide specific risk values it does provide a relative ranking of risks that provides risk management staff with a prioritized list of risks that allows for the most severe risks to be addressed first.

Practical risk management bases risk assessment on the identification and assessment of threats to the organization's assets. NIST SP 800-30r1 [22] defines a threat as any event or circumstance that can 
adversely affect organizational operations or knowledge assets. A threat source is an adversarial or non-adversarial source that could cause damage to a system by exploiting a vulnerability. A vulnerability is defined as a security weakness in the program or an asset's controls. Threat sources can be internal or external to the organization. In addition, threats can be intentional or accidental. Finally, a threat source can be a natural or man-made disaster. Threat assessment is about identifying threat sources and the vulnerabilities they can use to exploit/damage a knowledge asset. The purpose of threat assessments is to identify specific threat sources and the vulnerabilities that would be used to attack the organization's knowledge assets. The Risk Formula and assessment uses the results of threat assessment by using the consequence/damage caused by a threat utilizing a vulnerability and the probability/likelihood of this occurring to determine risk values/ratings.

To assist organizations in performing threat assessments generic threats are proposed. One such set is from Whitman and Mattord [33] who propose 12 threat categories:

- Compromises to intellectual property

- Deviations in quality of service

- $\quad$ Forces of nature

- $\quad$ Espionage or trespass

- Human error or failure

- Information extortion

- $\quad$ Sabotage or vandalism

- $\quad$ Software attacks

- Technical hardware failures or errors

- $\quad$ Technical software failures or errors

- Technical obsolescence

- Theft

The purpose of this list of threats is to provide organizations a guide as to where to look for knowledge security threats. It should be noted that this threat list includes technical threats, behavioral threats and legal threats. It is our purpose to propose such a list for KM/knowledge systems. Since $\mathrm{KM} /$ knowledge systems primarily focus on providing knowledge users with knowledge for making decisions our paper focuses on risks/threats associated with the capture, storage, retrieval, and use of knowledge. Our list considers technical, behavioral, and legal threats, and are generated by analyzing how KM/knowledge systems can be misused or abused with the specifics of how this is done being presented in the next section.

\subsection{Risk Assessment Frameworks}

The purpose of any risk assessment framework is to establish rules for what is assessed, who are the main actors that need to be involved in this assessment, creates terminology for assessment, criteria for quantifying, qualifying, and comparing degrees of risk, and provides a way to document all of this [20]. The main purpose of a risk assessment framework is to establish an objective measurement of risk so that an organization can understand risk and take appropriate action to mitigate it and bring it to an acceptable level.

Information technology professionals can choose from several risk assessment frameworks [20]: OCTAVE (Operationally Critical Threat, Asset, and Vulnerability Assessment) and NIST risk assessment framework, ISACA's Risk IT (part of COBIT), and ISO 27005:2008 (part of the ISO 27000 series). While these frameworks differ some in their prescription on how to do risk assessment, they all have the following five parts in common [5]:

- Development of risk measurement criteria

- $\quad$ Asset inventory

- Threat identification

- $\quad$ Risk score calculation

- Documentation of existing controls

- Identification of improvements to controls

Researchers and practitioners have been working on risk assessment frameworks for the area of KM/Knowledge Systems. These are discussed below.

\section{KM/Knowledge Systems Risk Assessment Frameworks}

Three KM knowledge system risk assessments have been proposed. Thalman et al. [29], Padyab et al. [26], and Ilvonen et al. [6] have all proposed $\mathrm{KM} /$ knowledge systems risk assessment frameworks that are differentiated by the $\mathrm{KM} / \mathrm{knowledge}$ contexts upon which they focus. Thalman et al. [29] focused on the knowledge sharing process context as the basis for assessing risk and then mapped the traditional information security framework into a KM security framework. Padyab et al. [26] also focused on the knowledge sharing process context and then used communication genres and the OCTAVE Allegro risk framework to create a $\mathrm{KM} / \mathrm{knowledge}$ system risk framework. Ilvonen et al. [6] expanded the context of their KM risk framework to include the knowledge processes of knowledge creation, knowledge sharing, and management of knowledge; and then fused it with the NIST SP 800-37r2 risk management framework to create their knowledge security risk management framework (see Figure 1). Ilvonen et al [6, p.13] define knowledge security "as the managerial process of organizations to identify threats toward important knowledge and secure the 
knowledge against those threats.” In the first stage, the business case of the intended KM initiative or change to the initiative, and a description of what kind of benefits are sought by the initiative/change are described by an organization. This is followed by knowledge identification that can be done in three ways: (1) examination of different locations, uses, topics and destinations of knowledge; (2) genrebased approach where different actors and their way of communication with other actors are identified and (3) examination of the knowledge systems where the knowledge resides. Threat identification happens along the lines of external threats and accidental or deliberate internal threats to the knowledge assets. In the risk analysis step, the identified threats are individually analyzed to recognize what kind of risks they may cause and how significant these risks are. Cost/benefit analysis deals with the costs of implementing controls that mitigate these risks. Step six, risk controls that need to be implemented are identified along with a plan for implementing them. Finally, monitoring which is a crucial part of Ilvonen et al [6] framework provides the organization with situational awareness of the risk environment the organization is facing.

We use the Knowledge Security Risk Management Framework as it uses a fuller context of how knowledge processes fit the overall $\mathrm{KM} / \mathrm{knowledge}$ systems business processes and is thus a more complete $\mathrm{KM} / \mathrm{knowledge}$ system risk management framework.

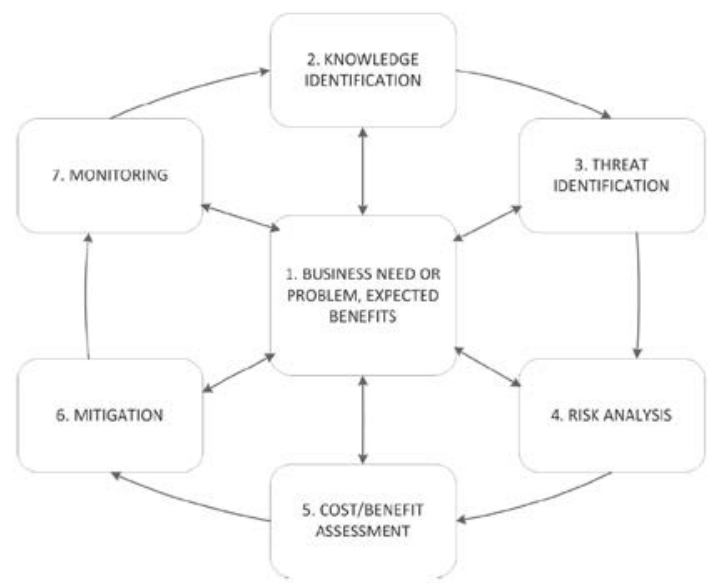

Figure 1 Knowledge Security Risk Management Framework [6]

\section{1. KM/Knowledge System Threat Assessment}

Figure 1 includes threat identification as a part of the risk management framework. To create a generic set of threats for $\mathrm{KM} / \mathrm{knowledge}$ systems we start with the basic $\mathrm{KM} /$ knowledge system processes of knowledge creation, sharing, and management and then add a further concern from Walsh and Ungson [31] who identified three contexts in which Organizational Memory (OM) could be misused:

- $\quad$ Automatic retrieval of knowledge may lead to a routine decision response when a nonroutine decision response is warranted;

- The controlled retrieval of knowledge may lead to a non-routine decision response when a routine decision response was appropriate;

- A controlled retrieval of knowledge may be appropriately activated in an attempt to elicit a non-routine decision response, but it may be implemented poorly.

Misuse of the OM occurs when organizational members self-servingly select knowledge to support positions that serve their political needs rather than the organization's [31]. Also, misuse of $\mathrm{OM}$ is a unique threat to knowledge use. These three misuse risks are also applicable to KM/Knowledge Systems as $\mathrm{OM}$ is a fundamental and integral part of $\mathrm{KM} /$ Knowledge Systems [16] and as such, OM threats are KM/Knowledge Systems threats.

We find the main $\mathrm{KM} /$ Knowledge Systems specific threats to be based on a threat analysis of the Walsh and Ungson [31] OM risk context above as well as by integrating traditional security threats (such as those listed by Whitman and Mattord [33]) to $\mathrm{KM} /$ knowledge system processes (creation, storing, sharing, and management) to generate the below set of $\mathrm{KM} / \mathrm{knowledge}$ system specific generic threats. This list reflects the misuse, abuse, disclosure, and loss of knowledge within the $\mathrm{KM} /$ Knowledge system and are grouped by technical, behavioral, and legal threats. Studies that illustrate or support the identified threats are also liste:

1. Failure to identify and capture critical knowledge in the knowledge creation process. This has been observed in several studies: Jennex and Zyngier [19] observed that large organizations that relied on humans to identify and capture critical knowledge often failed to sometimes identify and many more times capture critical knowledge. Jennex [10] [11] observed the failure to identify and capture critical knowledge in an engineering organization when identification of what to capture and to what detail to capture knowledge was left to organizational members with different levels of understanding the knowledge. In some cases, very experienced members failed to see the importance of capturing sufficient detail to make the 
knowledge useful to less experienced members; and having less experienced members misidentify critical knowledge because they did not know what was important. Finally, Jennex (2010, 2013) observed that many organizations failed to retain captured critical knowledge after a change in storage formats. Particularly observed was the loss of knowledge as organization migrated to newer versions or different word processing packages or to a newer storage media (example is moving from floppy disk drives to laser disks to USB drives). What was observed was that organizations failed to transfer all the knowledge from one format or package to the newer format or package resulting in a loss of knowledge that many times was found out later to be critical knowledge.

- Technical threats are from using automated tools to identify and capture critical knowledge with the vulnerabilities typically being in the ontologies and taxonomies used to guide the automated tools not accurately reflecting the domains being analyzed. Additional threats are from technical obsolescence leading to the loss of knowledge as the organization migrates to newer technologies.

- Behavioral threats are from personnel intentionally or accidently classifying critical knowledge and not recognizing critical knowledge, not capturing enough detail with the knowledge, or failing to capture it when it is recognized.

2. Disclosing critical knowledge to unauthorized recipients in the knowledge sharing processes. Jennex [10] [11] observed engineers sharing knowledge based on their own opinions of what was appropriate to share rather than following organizational guidelines. Jennex and Zyngier, [19] observed members of organizations sharing critical knowledge with unauthorized individuals because the organization had failed to classify disclosure categories of knowledge. Jennex [12] observed for crises and disasters the issue of what knowledge should be posted on social media and what knowledge should be trusted off of social media. Walters [32] documents the use of USB drives to capture knowledge from users who plug the drives into their system.

- Technical threats are from exploitation of communication vulnerabilities common to all communication systems and is focused on communication processes specific to $\mathrm{KM} /$ knowledge systems (as an example consider an organization using SharePoint as the system for communicating knowledge with the communication vulnerabilities being common to both knowledge and nonknowledge applications). Additional threats are from storage media by not properly securing access to cloud storage and/or server storage.

- Behavioral threats are from intentionally or accidentally failing to maintain access control lists for authorizing approved personnel to access knowledge, posting knowledge to inappropriate forums, not following disclosure processes, not encrypting knowledge in motion, falling victim to social engineering attacks and either disclosing knowledge to unauthorized individuals or allowing malware onto their systems that are collecting and transmitting knowledge to unauthorized individuals.

- Legal threats are from intentionally or accidentally not complying with disclosure laws such as those dealing with personally identifiable information or patient health knowledge. Other threats are from the intentional theft of intellectual property and from the unintentional loss of intellectual property based on the cloud or collaborative medium on which it was stored or shared [27].

3. Losing critical knowledge by not capturing it from critical human sources. Jennex [15] and Jennex and Durcikova [17] observed this as an issue and proposed a process for rating knowledge sources and their likelihood of departing as well as providing a score to indicate the time criticality of taking action.

- Behavioral threats are from intentionally or accidentally not identifying critical human knowledge repositories and taking actions to capture and store the critical knowledge (this threat is typically not capturing knowledge from retiring personnel but also can occur by not capturing knowledge from personnel departing an organization for reasons other than retirement).

- Legal threats are from intentionally or accidentally not complying with required knowledge capture (an example of this was Nuclear Regulatory Commission requirements on nuclear stations to capture critical knowledge from employees prior to large scale work force layoffs).

4. Losing critical knowledge by not storing it on nonvolatile media or by not migrating knowledge with changing storage standards. 
Jennex, [12] [14] has reported large scale losses of critical knowledge due to storage media not surviving as long as expected. Losses include NASA losing plans for moon craft, nuclear plants losing documents stored on older storages media such as $8,5 \frac{1}{4}$, and $3 \frac{1}{2}$ inch floppy disks, microfiche, paper, etc. Additional knowledge losses were observed as organizations migrate to newer softwares or newer versions of software, and changing data storage formats.

- Technical threats are from the failure of storage media, hardware, and/or software.

- Behavioral threats are from intentionally or accidentally not following technology procurement processes, selecting providers without checking their technology, not planning for obsolescence, not testing technologies before applying them or while using them, and/or artificially obsoleting technologies before age requires it.

5. Giving bad advice by not using appropriate knowledge. This risk is illustrated by high profile errors in decision making such as Jennex [13] report on the conviction of the scientific panel who told citizens there was little likelihood of a seismic event prior to the 2009 L'Aquila, Italy earthquake that killed 309. Vaughn [30] and Boin and Fishbacher-Smith, D. [4] discuss the decision making process that ignored safety concerns from the contractors prior to the 1986 Challenger space shuttle disaster. Perrow [25] discusses the human errors made that led to the severity of the 1979 Three Mile Island nuclear disaster

- Technical threats are from search tools not finding relevant knowledge, improperly prioritizing knowledge, not using integration tools allowing relevant knowledge to not be incorporated into search results, and/or using visualization technologies that influence decision makers to the wrong option.

- Behavioral threats are from decision makers using incomplete knowledge, and/or inappropriately applying knowledge to unsuitable decision contexts.

- Legal threats are from decision makers not utilizing due care or due diligence in assessing knowledge.

\section{Newly Proposed Risk Assessment Framework for Knowledge Management/Knowledge Systems}

Ilvonen, et al. [6] proposed a risk management framework for $\mathrm{KM} / \mathrm{knowledge} \mathrm{systems} \mathrm{as} \mathrm{shown} \mathrm{in}$ Figure 1. We propose an enhancement to this framework that offers a high-level KM risk assessment. Jennex [15] proposed a risk management process for assessing knowledge loss risk with departing personnel. This was a form based process that walked managers through the risk assessment process. Jennex and Durcikova [17] utilized this form based risk assessment process to assess the knowledge loss risk with graduate students and found the form based process to be a good process. Based on this experience and further literature review provided on both general risk assessment frameworks and also on $\mathrm{KM} /$ Knowledge Systems framework, we are proposing the following template for knowledge risk assessment [5]. The definitions and possible values of each category are provided in Table 1.

In general terms, an asset can be people, knowledge, processes, systems, or applications. We propose that the key asset for $\mathrm{KM} / \mathrm{knowledge}$ systems is the knowledge from a particular expert or knowledge worker.

When creating the risk assessment for $\mathrm{KM} / \mathrm{knowledge}$ systems, one would identify all the different knowledge assets and then identify every threat to each knowledge asset, following the categories in Table 1. In general, the impact areas are one or several from the following: financial (expenses and revenues), productivity (business operations), reputation (perceptions of customers and society), legal (fines and litigations), and safety (safety or employees, customers, environment). It is important to note that these areas are not exhaustive, and that an organization may remove or add areas. The importance of each area must be ranked as low/medium/high while keeping in mind that not all areas are high impact. Next, the impact of each threat on the asset must be in each are must be evaluated. For example, business leaders must evaluate whether a loss of $1 \%$ or $5 \%$ of revenue is considered to have a low/medium/high impact. Scenarios must be written out so that anybody who reads them can follow the logic or the arguments. The next step is to calculate the risk score that is the product of the ranking and impact. This score is meaningless by itself, but very useful when we compare the impact of different threats on different assets.

An organization can pick from one to four risk control strategies: accept (taking no action against the treat), mitigate, share, or defer. The only threats that should be accepted would have a very low risk score. Most of the threats will be mitigated (control(s) would be implemented) or shared (e.g., insurance 
would be purchased). Examples of control could be codification of knowledge that would happen on a regular basis either by automatic agents or by apprentices who shadow a knowledge worker.

In congruence with the traditional risk assessment frameworks, the highest risk score threats must be processed as first and appropriate controls must be initiated.

\section{Contribution to Research and Practice}

Creation, storage, and reuse of knowledge is a source of both short term and long term competitive advantage for organization [1]. Thus, organizations need to pay more attention to safeguard this important asset.

Our contribution to research builds on existing generic risk assessment frameworks and knowledge management frameworks to create an easy way to calculate the risk score for each threat to an asset. Specifically, we contribute to research in the following ways: First, we provide an in-depth discussion of threats to knowledge assets (see section 3.1). Nor the generic framework or the knowledge management security risk assessment provide such a guideline. Second, we provide a concrete approach on how to calculate the risk score by identifying possible impact areas, rank the importance of these impact areas, easily score the impact. While the risk score by itself is not that useful, it becomes very useful when compared to risk scores of other threat/asset pairs.

Our contribution to practice is twofold: First, we provide a business-driven approach to identifying and categorizing (low/medium/high) the impact of a threat for five business area - financial, productivity, reputation, legal, and safety. The impact needs to be discussed with managers as they are the best informed what is a high/low impact. Second, our approach offers a use of a template that can be followed by both technical and non-technical staff and thus allows for a faster documentation of assets/threats, their potential impact, and risk mitigation techniques.

Table 1 Risk Assessment for Knowledge Management

\begin{tabular}{|c|c|}
\hline Asset & $\begin{array}{l}\text { The object of the risk assessment - knowledge from particular } \\
\text { expert or knowledge worker }\end{array}$ \\
\hline Asset Owner/Custodian & $\begin{array}{l}\text { Owners have the authority to accept risk. Custodian of the asset } \\
\text { are responsible for implementing and maintaining controls that } \\
\text { protect the asset. }\end{array}$ \\
\hline Asset Importance & Low/Medium/High \\
\hline Threat & $\begin{array}{l}\text { Any way an asset could be compromised that would have an } \\
\text { impact on a business. See examples in the Knowledge } \\
\text { Management/Knowledge System Threat Assessment section of } \\
\text { this paper. }\end{array}$ \\
\hline Threat Description & $\begin{array}{l}\text { Failure to identify and capture critical knowledge in the } \\
\text { knowledge creation process. } \\
\text { Disclosing critical knowledge to unauthorized recipients } \\
\text { in the knowledge sharing processes } \\
\text { Losing critical knowledge by not capturing it from } \\
\text { critical human sources. } \\
\text { Losing critical knowledge by not storing it on } \\
\text { changolatile media or by not migrating knowledge with } \\
\text { Giving bad advice by not using appropriate knowledge. }\end{array}$ \\
\hline Likelihood & Low (1)/ Medium (2)/ High (3) \\
\hline Impact on & $\begin{array}{l}\text { Confidentiality of knowledge } \\
\text { Integrity of knowledge } \\
\text { Availability of knowledge }\end{array}$ \\
\hline
\end{tabular}




\begin{tabular}{|l|l|l|l|}
\hline Impact Area & Ranking & Impact & Score \\
\hline Financial & $\begin{array}{l}\text { Low (1)/Medium (2)/High } \\
(3)\end{array}$ & Low (1)/Medium (2)/High (3) & \\
\hline Productivity & $\begin{array}{l}\text { Low (1)/Medium (2)/High } \\
(3)\end{array}$ & Low (1)/Medium (2)/High (3) & \\
\hline Reputation & $\begin{array}{l}\text { Low (1)/Medium (2)/High } \\
(3)\end{array}$ & Low (1)/Medium (2)/High (3) & \\
\hline Legal & $\begin{array}{l}\text { Low (1)/Medium (2)/High } \\
(3)\end{array}$ & Low (1)/Medium (2)/High (3) & \\
\hline Safety & $\begin{array}{l}\text { Low (1)/Medium (2)/High } \\
(3)\end{array}$ & Low (1)/Medium (2)/High (3) & \\
\hline & & $\begin{array}{l}\text { Risk Score } \\
\text { (Likelihood x Impact Score) }\end{array}$ & \\
\hline Adequacy of Existing Controls & Low/Medium/High & & \\
\hline Risk Control Strategy & - Accept & & \\
\hline Ritigate & & \\
\hline Control 1 & - Share & & \\
\hline Control 2 & Description & & \\
\hline
\end{tabular}

\section{Conclusion}

This paper builds on previous generic risk assessment framework and the Knowledge Security Risk Assessment Framework [6] to create the risk assessment for knowledge management. This addition to the framework contributes to the current research and practice, by providing a $\mathrm{KM} / \mathrm{knowledge}$ system specific threat analysis and a template that can be followed to not only capture the knowledge asset, but also to capture the potential threat, it's likelihood, impact, risk score in an easy to understand fashion that streamlines the whole process. Previous research that discussed risk in KM did not provide such a step-by-step approach that is repeatable in any organizations.

Future research will apply the framework in case studies, preferably in organizations that have previously performed risk assessments so that the results from that risk assessment and the risk assessment performed using our proposed threat analysis and risk form can be compared.

\section{References}

[1] Alavi, M., \& Leidner, D. E. (2001). Knowledge management and knowledge management systems: Conceptual foundations and research issues. MIS quarterly, 107-136.
[2] Aubert, B., M. Patry, and Rivard, S. (1998). “Assessing the Risk of IT Outsourcing," Proceedings of the 31st Hawaii International Conference on Systems Sciences. IEEE Publishing, 685 - 693.

[3] Billington, J. (1997). "A Few Things Every Manager Ought to Know About Risk," Harvard Management Update, 2(3), March, pp. 1-12.

[4] Boin, A., \& Fishbacher-Smith, D. (2011). The importance of failure theories in assessing crisis management: The Columbia space shuttle disaster revisited. Policy and Society, 30(2), 77-87.

[5] FitzGerald, J., A. Dennis, A. Durcikova, "Business Data Communications and Networking," Wiley, 13th Edition (2017)

[6] Ilvonen, I., Jussila, J. J., \& Kärkkäinen, H. (2015). Towards a business-driven process model for knowledge security risk management: Making sense of knowledge risks. International Journal of Knowledge Management (IJKM), 11(4), 1-18.

[7] ISO 27001:2013 (reviewed 2019) Information technology -- Security techniques -- Information security management systems - Requirements, International Standards Organization. 
[8] ISO/IEC 27005:2018 Information technology -Security techniques -- Information security risk management, International Standards Organization

[9] Jennex, M.E., (2010). "Preface: Why Knowledge Management?" “Ubiquitous Developments in Knowledge Management: Integrations and Trends," Edited by Murray E. Jennex, Information Science Reference, Hershey, PA, pp. xviii-xxix.

[10] Jennex, M.E., (2008). “Impacts From Using Knowledge: A Longitudinal Study From A Nuclear Power Plant,” International Journal of Knowledge Management, 4(1), pp. 51-64.

[11] Jennex, M.E., (2008a). "Exploring System Use as a Measure of Knowledge Management Success,” Journal of Organizational and End User Computing, 20(1), pp. 50-63. Jennex, M.E., (2013). “Knowledge Management: The Risk of Forgetting." iKNOW, the magazine for Knowledge Workers, 3(1), pp. 4-7.

[12] Jennex, M.E. (2010). "Implementing Social Media in Crisis Response Using Knowledge Management.” International Journal of Information Systems for Crisis Response and Management, 2(4), pp. 20-32.

[13] Jennex, M.E., (2012). "Risk and Reward in Crisis Response." The International Journal of Information Systems for Crisis Response and Management, 4(3), pp. iiii.

[14] Jennex, M.E., (2013). "Knowledge Management: The Risk of Forgetting.” iKNOW, the magazine for Knowledge Workers, 3(1), pp. 4-7.

[15] Jennex, M.E., (2014). "A Proposed Method for Assessing Knowledge Loss Risk with Departing Personnel” VINE: The Journal of Information and Knowledge Management Systems, 44(2), pp. 185-209.

[16] Jennex, M.E.; Dittes, S.; Smolnik, S.; Croasdell, D.; and King, D., (2018). Knowledge, Innovation, and Entrepreneurial Systems at HICSS. Communications of the Association for Information Systems: Vol. 43, Article 39. DOI: 10.17705/1CAIS.04339, Available at: https://aisel.aisnet.org/cais/vol43/iss1/39

[17] Jennex, M.E. and Durcikova, A. (2013). “Assessing Knowledge Loss Risk.” 46th Hawaii International Conference on System Sciences, HICSS46, IEEE Computer Society, January 2013.

[18] Jennex, M.E. and Durcikova, A. (2014). "Integrating KM and Security: Are We Doing Enough?” International Journal of Knowledge Management, 10(2), pp. 1-12.

[19] Jennex, M.E. and Zyngier, S., (2007). "Security as a Contributor to Knowledge Management Success" Information Systems Frontiers: A Journal of Research and Innovation, 9(5), pp. 493-504.
[20] Mackey, R., (2019). “Choosing the right information security risk assessment framework" Techtarget.com, retrieved June 4, 2019 from https://searchsecurity.techtarget.com/magazineContent/Info rmation-security-risk-assessment-frameworks

[21] NIST SP 800-12 rev 1, (2017). An Introduction to Information Security, National Institute of Standards and Technology, retrieved June 8, 2019 from https://nvlpubs.nist.gov/nistpubs/SpecialPublications/NIST. SP.800-12r1.pdf

[22] NIST SP 800-30 rev 1, (2012). Guide for Conducting Risk Assessments. National Institute of Standards and Technology, retrieved June 8, 2019 from https://csrc.nist.gov/publications/detail/sp/800-30/rev$1 /$ final

[23] NIST SP 800-37 rev 2, (2018). Risk Management Framework for Information Systems and Organizations: A System Life Cycle Approach for Security and Privacy, National Institute of Standards and Technology, retrieved June 8, 2019 from https://nvlpubs.nist.gov/nistpubs/SpecialPublications/NIST. SP.800-37r2.pdf

[24] NIST SP 800-53 rev 5 (Draft), (2017). Security and Privacy Controls for Information Systems and Organizations, National Institute of Standards and Technology, retrieved June 8, 2019 from https://csrc.nist.gov/publications/detail/sp/800-53/rev5/draft.

[25] Perrow, C. (1981). Normal accident at three mile island. Society, 18(5), 17-26.

[26] Padyab, A. M., Päivärinta, T., \& Harnesk, D. (2014). Genre-Based Approach to Assessing Information and Knowledge Security Risks. International Journal of Knowledge Management, 10(2), 13-27.

[27] Phelps, M. and Jennex, M.E., (2015). “Ownership of Collaborative Works in the Cloud,” International Journal of Knowledge Management, 11(4), pp. 35-51.

[28] Smith, H.A., McKeen, J.D., and Staples, D.S., (2001). Risk Management in Information Systems: Problems and Potentials. Communications of AIS, 7(Article 13).

[29] Thalmann, S., Manhart, M., Ceravolo, P., \& Azzini, A. (2014). An integrated risk management framework: measuring the success of organizational knowledge protection. International Journal of Knowledge Management (IJKM), 10(2), 28-42.

[30] Vaughan, D. (2004). Theorizing disaster: Analogy, historical ethnography, and the Challenger accident. Ethnography, 5(3), 315-347. 
[31] Walsh, J. P., and Ungson, G. R. (1991). Organizational Memory. Academy of Management Review, 16(1), pp. 57-

91.

[32] Walters, P. (2012). The risks of using portable devices. Carnegie Mellon University. Produced for US-CERT, a government organization. Retrieved from http://www. uscert. gov.

[33] Whitman, M.E. and Mattord, H.J. (2019). Management of Information Security, 6th edition. Course Technology, Boston, MA. 